\title{
Knowledge Intensive Computer Aided Design: Past, Present and Future
}

\author{
Tetsuo Tomiyama ${ }^{1}$ and Ken Ping $\mathrm{Hew}^{2}$ \\ 'RACE, The Univeristy of Tokyo, Japan \\ ${ }^{2}$ JSPS-RFTF-The Modeling of Synthesis Project, The Univerity of Tokyo, Japan
}

Key words: Knowledge Intensive CAD, Knowledge Management, Life Cycle Knowledge

\begin{abstract}
This paper is a report of the past, present and future research in knowledge intensive computer aided design (KIC). This paper reports the two previous workshops on KIC and provides the discussions and conclusions reached by the present KIC workshop (KIC-3). Three working groups and one invited paper that were formed from the attendees at KIC-3 are briefly discussed. The groups were assigned to discuss knowledge life cycle, ontology, knowledge intensive design (KID) and knowledge representations. Finally, we provide some guideline for the future research in KIC. It discusses the challenges and opportunities involved in KIC research, such as strategy and technology of knowledge management, system implementation for integration under design support systems, practical applications and evaluation of KIC, and life cycle knowledge as the focused content of KIC.
\end{abstract}

\section{INTRODUCTION}

Computer Aided Design (CAD) technology plays a key role in today's advanced manufacturing environment. To reduce the time to market, achieve zero defect quality at the first production, and use available production and logistics resources effectively, product and design process knowledge covering the whole product life cycle must be used throughout product design. Once generated, this intensive design knowledge should be made available to later life cycle activities. Due to the increasing concern about 
global environmental issues and rapidly changing economical situation worldwide, design must exhibit high performance not only in quality and productivity, but also in life cycle issues, including extended producer's liability.

This requires designers and engineers to use various kinds of design knowledge intensively during product design and to generate design information for use in later stages of the product life cycle such as production, distribution, operation, maintenance, reclamation, and recycling. Therefore, future CAD systems must incorporate product and process knowledge which are not explicitly dealt with in the current systems, in their design tools and design object models. This paper reports the past, present and future research in KIC. We outline the key discussions and conclusions reached in the KIC-3 workshop.

\section{THE PAST}

Between 1987 and 1989, the IFIP Working Group 5.2 organized a series of three workshops on "Intelligent CAD," which were followed by a working conference on the same subject in 1991 (Yoshikawa et al., 1989, 1990, 1991, Brown et al., 1992). This new series of workshops extends the concept of intelligent CAD to the concept of "knowledge intensive engineering." Knowledge Intensive CAD has multiple type of knowledge regarding products and design processes. The concept advocates that intensive life cycle knowledge regarding products and design processes must be incorporated in the center of the CAD architecture. The concept focuses on the systematization and sharing of knowledge across the life cycle stages and organizational boundaries. It concerns product knowledge management, formalization and sharing knowledge across the life cycle stages and organizational boundaries. KIC workshops aim at clarifying and elaborating the concepts of knowledge intensive engineering and CAD by providing an international forum for mutual discussion and exchange of opinions of experts of the field.

The first IFIP Working Group 5.2 Workshop on "Knowledge Intensive CAD" (KIC-1) was held at Helsinki University of Technology, Finland, on 26-29 September 1995. KIC-1 was the first workshop that extended the concept of intelligent CAD to the concept of "knowledge intensive engineering." This workshop discussed a variety of issues related to KIC; knowledge intensive CAD frameworks, product and design process modeling, and methodologies, tools and techniques for knowledge intensive $C A D$. The result of the first KIC workshop appeared as a book in 1996 (Tomiyama et al., 1996). 
The second workshop (KIC-2) in the series was held at Carnegie Mellon University in Pittsburgh, PA, USA, on 16-18 September 1996. This workshop dealt with the issues in system architectures, representations and delivery systems, as well as addressing topics from the point of view of research achievements, research issues and industry needs. The result of the second KIC workshop appeared as a book in 1997 (Mäntylä et al., 1997).

The third workshop (KIC-3) in the series was held in Tokyo, Japan, on 47 December 1998. Based on the achievements made in the previous two workshops, it was aimed at identifying critical issues in ontology, KIC and knowledge intensive design, and knowledge representations. These issues later formed three working groups for the KIC-3 workshop. This paper reports the key discussions and conclusions made by these three working groups that were formed from the attendees at the KIC-3 workshop. The workshop's papers presented the current achievements and research issues in KIC.

\section{CURRENT ACHIEVEMENTS AND RESEARCH ISSUES IN KIC-3}

The section is about the current achievement using the discussion results from the KIC-3 workshop. The workshop has all together seventeen papers and one invited paper. Thirteen papers were accepted for KIC-3 volume after a review. The workshop was assigned into three discussion groups: ontology, knowledge intensive design and knowledge representation, and a keynote paper discusses knowledge life cycle. Key discussions from the invited paper and discussion groups are presented as below.

\subsection{Knowledge Life Cycle}

The present industry is focused on increasing the volume of projects and customers (and, therefore, revenue), reducing costs and shortening the delivery time and product life cycle. These are the forces that drive the companies throughout the world investigating, assessing, and reengineering their operations to reduce risk, to increase productivity, to streamline the design processes, and to improve their performances through automation and information technology. Effective management of life cycle knowledge is a key element in these activities. In the future, in order for the companies to keep the excellency of business, they must "reuse of existing knowledge" and make sure all knowledge created or learned is just-in-time for later use 
in a correct, accessible, and trusted manner from the very early stages of design (Mäntylä, 1996).

Blessing and Wallace introduce a conceptual system that supports the knowledge life cycle called process-based support system (PROSUS). In the PROSUS system, the flow of knowledge life cycle is accessible, relevant and trusted by the designers. The authors give an observation about the problem of mismatch between the available information resources and the sources of information that are accessed by designers. The authors give a discussion on the challenge of KIC development to capture this life cycle knowledge and its context, in the way that knowing where and when to apply it, as this knowledge constitutes expertise and is needed to determine relevancy and to build trust. The authors first characterize the intensive knowledge in engineering design as wide variety of issues, complex relationship between issues, and dynamic nature of the knowledge. The later focuses their work, in particular on capturing the knowledge that is being created and used in a project, as and when it is being created or used. Design Matrix is used as an approach to group together various types of design issues and life cycle knowledge. The most important contribution of this work is the new way of capturing and indexing life cycle knowledge based on the context as and when it is generated, and make it immediately available to other project members.

\subsection{Ontology}

Current CAD systems support complex tasks but they do not possess the body (intended meaning) of knowledge necessary for generating adequate design solutions. The designer has to take this burden. This burden should be shifted as much as possible back to the CAD systems. Ontology is a promising candidate to help to achieve this. Thus, ontology is a tool that captures the intended meaning of concepts and statements in a domain. Ontology has been studied and developed in artificial intelligence and have been proposed as a specification mechanism for knowledge sharing and reuse across different applications in KIC. A better understanding both of its roles in KIC and of its complex problems was discussed in KIC. Three papers focused on ontology issues in KIC have been presented at the KIC-3 workshop.

Salustri gives a discussion on formalizing a language for a product model considering the basis of the concept of "ontological commitments." The author argues why a totally new representation language is needed. He gives an explanation on how the "ontological commitments" can be constructed to map logical structures to the domain of design knowledge. He then goes on 
to explain how the commitments are necessary to establish a language for product modeling.

Sekiya, Tsumaya and Tomiyama give a discussion on formalizing structure of knowledge in an integrated manner for a modeling process. They present a Knowledge Intensive Engineering Framework to analyze and validate a design object by integrating various computational tools such as Finite Element Analysis (FEM) and its knowledge. The authors break the design of a FEM model in four stages: a design stage, where the phenomena to be studied via FEM are extracted and identified; an analysis stage, where relevant parameters of the artifact being studied are selected; modeling theory stage, where the artifact and FEM-related knowledge are combined to select a focus for the analysis and choose an appropriate analysis method; and tool stage where a FEM network is generated. The most important contributions of this work are the representation of designer's thought process and the formalization of knowledge in model building.

Shah, Rangaswamy, Qureshi and Urban present a system based on an ISO/STEP compatible data model that has two components: a product and process model. The system aims at supporting the design process by acting as a design history system. The authors concentrate on models of design rationale and suggest implementation using STEP-related tools. The most important contributions of this work are the information model of design rationale and the demonstration that it can be mapped to implementation structures to create a prototype design information capture system.

\subsection{KIC and Knowledge Intensive Design}

It was not until the mid-1990s that intensive knowledge started to be introduced into $\mathrm{CAD}$, and since then new CAD theories and design concepts have been introduced in line with knowledge management results. KIC is a field of study that focuses on developing computational techniques for performing complex design tasks. The designed product of these processes takes advantage of intensive knowledge flows at different stages. One of the important aspects of addressing intensive knowledge for CAD is to create a design tool that would effectively make reuse of product information at many levels, starting with the functional specifications and overall design rationale and ending with individual product modules, components, and their technical and geometric details. Seven papers were included in this workshop and they presented the most up to date concepts of KIC and knowledge intensive design (KID). Papers of Xue, Ozawa et al., Rosen, and Yoshioka et al. discuss the issue of KIC and papers of Susca et al., Chan et al., and Bok et al. discuss the issue of KID. 
Xue discusses the issues in modeling product life cycle knowledge and data for an intelligent concurrent engineering design system. The author reports on the design and implementation of a concurrent design system that maintains several aspects of a design under development using AND-OR tree structure and feature concepts. The approaches suggested provide a basis for building a KIC system with concurrent design capabilities.

Ozawa, Cutkosky and Howley give an overview of the work done on an agent-based concurrent engineering system and model sharing in a collaborative CAD. The focus of this paper is the development of methods for capturing and exchanging the information that agents need to share in a machine-readable form. The authors first review the design information sharing problem and examine the different levels of abstraction and detail at which such information is represented and shared. They then introduce a representation language and information-sharing infrastructure that was employed in the agent-based concurrent engineering system. The authors describe how this approach can be used to redesign an optical pick-up head for DVD (digital versatile disk) player. The benefits using this approach facilitate (design) model sharing and execute more design tasks in parallel and evaluate design trade-off from the very early design stages.

Rosen discusses integration of product realization processes in RTTB, a system based on decision-based design, which is an approach using explicit problem-solving models created separately by "meta-design" activities. The author also discusses a typical prototyping case study.

Yoshioka, Shamoto and Tomiyama describe the use of KIEF (this concept already appeared and discussed in the previous KIC workshops) environment in a new domain of building foundation design and engineering. The authors usefully illustrate a knowledge intensive CAD system that is capable of describing the casual relationships among physical phenomena and relationships between physical phenomena and analysis methods.

Susca, Mandorli, Rizzi and Cugini describe an application of a commercial knowledge-based configurator to the problem of designing a racing car in a limited problem domain. The authors use knowledge-based engineering technology to represent design information of a car bodyweight. The information related to design of a car body, such as design specifications, engineering rules, component shapes and design constraints, is captured in a knowledge-based system. This information is arranged in a polymorphism manner that the digital car model can actually respond in different ways and take many forms driven by the configuration values.

Chan and Finger introduce the concept of "reflective-interactive" design aiming at representing not only the artifact being designed, but also designer's "situation" with respect to the design process, i.e. the design 
context. They suggest a design artifact model composed of "concepts" and "relationships"; concepts are composed of "phases" related to function, behavior, and form. A rough proof-of-concept implementation is also outlined.

Bok, Myung, Han, Moon and Park describe an experimental system that permits a connection between a commercial CAD system (Solid Edge) and an expert system shell to be created across Internet using distributed object technologies (OLE/DCOM and COBRA). The authors give useful details on the implementation of a KIC system to support commercial product design.

\subsection{Knowledge Representation for KIC}

Knowledge representation (KR) is a set of syntactic and semantic conventions for describing designer's know-how information. KR syntax specifies on symbols used and the ways they arranged. The study of semantics in KR is mainly focused on how the meaning is attached to the syntax. The sorts of things what need to be represented are design problems, knowledge and reasoning and range from objects, (sequences of) design processes/events, human know-how and what we do not know (human learning). For reasoning, the representations are focused on deductive (inferring new knowledge from old), inductive (inferring generalization from known instances), by analogy (inferences based on similarities), and metareasoning (inferences based on meta-knowledge; knowledge about decision making strategies and knowledge about information/raw data). We believe that to create and use a large-scale domain theories with multiple axioms will require concepts such as machine learning, advanced reasoning methods and integrated life cycle knowledge media and framework. Two papers were selected to discuss the above issues and they present the current state-of-the-art of KR in KIC.

Crecu and Brown focus on machine learning in a group of collaborative agents. They claim that agents in CAD have to display flexibility in learning like a human. They usefully discuss the need for learning, the parameters of learning, where and when to learn, and the possibility of refocusing on new learning tasks essential for multi-agent design systems. A method of guiding learning in multiple agents design systems is described. The basic idea in this method is to use deviations of "expectations" that agents have on each other to trigger a learning process. The learning itself is based on a "wrapper" that tries to choose the best way to maintain expectations so that unexpected responses of other agents can be accommodated. 
Borg, Yan and Juster describe a system that supports the designer by providing knowledge about consequences of the decisions they take. These consequences cover the whole product life cycle. Their work deals with concepts, architecture, implementation and initial experience with a KIC system intended to offer a design environment that can provide a designer with pro-active help about life cycle consequences of design choices.

\section{THE FUTURE DIRECTIONS}

KIC research and development have undergone considerable changes and improvements over the last three years. KIC has become more complex using new technological developments, well-structured ontology, and better knowledge representation strategies. However, from the viewpoints of practical developments, there are also increasing competition, stronger customers' awareness and stricter regulations. This means that technical products and processes also become complex at the same time. Products have to be technically excellent in design as well as commercially successful. All of these have resulted in better quality, better value for money, shorter time to market and tighter requirements.

New directions to improve effectiveness and efficiency of KIC research and development are needed to cope with these changes and to support the product development in order to remain competitive. The basic needs of industry are making products better, faster, cheaper and meet all the safety regulations. According to Brown (1997), these needs remain the same in the last ten years and future KIC systems have to "satisfy the needs of industry" as one of the goals. There is a new recognition and need that the design process must consider; an effective knowledge management and knowledge life cycle, that can be examined and refined together with the KIC architecture. Knowledge is the key resource that is important to capture, maintain, improve and use within the KIC system. The problem and issue of knowledge management and implementation in KIC are complex. They must be understood both from the practical and the theoretical perspectives. The followings are some research directions that represent various combinations of the practical and theoretical aspects.

\subsection{Strategies and Technologies for Knowledge Management}

The overall aim of knowledge management (KM) is to translate knowledge into value. Technology is, of course, important. Without it, KM 
will be difficult to implement. This is a good reason to establish a better KIC technology for the future. It is arguable that businesses can only survive and thrive by exploring every possible advantage in an increasingly competitive market. The knowledge that an organization might have range from company's intellectual property (know-how, know-what, know-why and know-who) to the special skills and innovative engineering design processes. These are the assets worth capturing, keeping, sharing and nurturing. Here, the focus of KIC technology is to take a hard look at these sets of assets as the challenges and opportunities that drive the entire engineering design and business process towards better performances and best practices. New KIC research directions must consider the combination of $\mathrm{KM}$ with multiple disciplinary designers, strategies and technologies. Future KIC systems have to share knowledge to gain competitive advantages and also to be capable of feeding knowledge from those who have it to those who need it.

The above discussion raises a number of important questions for future KIC development within the scope of KM:

i. How to systematize knowledge?

ii. How to manage knowledge systematically?

iii. How to share knowledge systematically?

iv. How to translate knowledge into value systematically?

v. How to use knowledge?

vi. How to continually absorb and renew knowledge in a KIC system?

vii. How is a successful knowledge management established for a KIC system?

viii. What are the strategic problems that could be solved if knowledge about better performance and best practices were better managed, shared, translated and used systematically within a KIC system?

The first to forth questions lead to issues such as what techniques should be used to manage, share, translate and use knowledge, and what tools can be used to computerize this knowledge structure systematically. The techniques and tools must have these criteria: (i) a shared knowledge representation (language and vocabulary), (ii) protocols that can represent notification and subscription and exchange information, (iii) facilities for context and content-directed routing, and (iv) intelligent matching of information for getting knowledge from "producers" and delivering to "consumers" who need it. Merely archiving knowledge in itself is inadequate; knowledge has to be improved and this is learning. The sixth question leads to issues as what type of machine learning technique can be used to keep up-to-date knowledge systematically. The seventh question leads to issues such as what the influences and validations of successes are how these influences correspond to the engineering design's objectives, and 
how they are validated or measured. Validation is needed to determine whether our research has been successful. Investigation into these issues will increase our understanding of design as a knowledge-intensive process and that knowledge has to be managed to facilitate the entire design process. The last question gives rises to issues related to the translation of this understanding into new KIC methods and theories considering the KM's elements. It also focuses on tests about the impact of the adopted techniques and tools. The traditional KIC research must be improved to resolve KM issues.

\subsection{Implementation of KIC Systems}

Future research and development should consider the implementation of the concept of KIC. It is the essential stage in the development of KIC in which the conceptual frameworks, knowledge representation and knowledge-intensive design are modeled in a computer in an executable form, such as programming language or mathematical method. Many current research efforts have focused on giving a systematic conceptual framework and discussed the architectural of the KIC system. Future research should use it to develop a computer-based system in order to test the concept and the feasibility of the methodology. The test is performed by programming a KIC conceptual framework and following this with a computer simulation to implement the codes as a working system, which will facilitate the design.

According to Hew (1998), there are four key steps and checklists in setting up a strategy for KIC implementation using advanced technology. They are outlined below.

Step I: Define the purpose (ultimate design goal) and policy of a KIC system. The first step is to identify the purpose of the system, and the policy about how much information and knowledge to allocate into the system in order to meet the final design goal or business plan. Checklist: Have a plan. The plan is important to address a design strategy and find out the application of KIC techniques necessary for business and design goals. Without it, the system developer will simply waste the technology, investment and time. The plan should be a constantly evolving procedure taking account of all details of early design stages input, barriers and objections. Some of the related information and knowledge to be worked out:

i. Basic aim, expressed in term of what the overall functions of KIC system are.

ii. Scope, expressed in term of what the content and context of the knowledge for the KIC system are. 
iii. Demand, expressed in term of how the designer would use the KIC system.

iv. Interactive multiple disciplinary knowledge resources for the design process.

v. Design constraints and integration, expressed in term of what the system can do and what it cannot do.

vi. Programming cost, time deadline, computer technology issues and modeling technique.

Step II: Re-design (program) the product in the KIC system. The second step is to know what the design scenarios, desired design activities, design functions, behaviors and states of the model are. It is also necessary to identify the integrated design methods for the multiple models in KIC considering the design scenarios, design activities, functions, behaviors, and states. Checklist: Keep it simple. KIC implementers should consider a simple solutions and systems for a complex design. Simply, the user has to be able to understand it. Some of the related information and knowledge to be work out:

i. What are the design activities, modules, design scenarios, functions, behaviors and states?

ii. Who is the user?

iii. How to divide each design activity, design scenarios, functions, behaviors, states into computer-based models in KIC and how many?

iv. How will the models be run and organized together, so that they can be interactively related each other?

v. What prototyping techniques and communication tools are required to program KIC and its design models?

Step III: Integrate knowledge using a synthesis and analysis design thought process. The third step is to consider an integrated design environment for KIC. Design is an interactive design process involving multiple disciplinary knowledge. It is important to identify a thought process to operate the knowledge in this interactive environment. According to Tomiyama (1998) and Hew et al. (1999), design knowledge operation can be systematically and mathematically defined by referring to analysis oriented and synthesis oriented thought processes. Elements of design knowledge that need to be interactively related and operated from one to another are knowhow, rules, standards, design requirements and anticipated physical needs for the environment (such as stiffness, energy consumption, maintenance, green and environmental issues, etc.), down to the smallest conceivable detail. Checklist: Plan top down, but implement bottom up and avoid overintegration. Implementation as an inside-out or bottom-up approach builds from the smallest knowledge elements to form a larger component of the 
product. There must be a trade-off between flexibility and integration, in particular, it should not replace the creativity of designer, but support the designer with useable knowledge.

Step IV: Implement KIC techniques and solutions to achieve Step I's purpose and policy. At this stage, a KIC system will be implemented module by module, or model by model, and in stages. KIC is implemented only if it is needed to achieve business needs and engineering design goals. Checklist: Be committed. A move towards the development of a "good engineering design assistant system" for the design process is needed. It is important to believe that such assistance is achievable. Support from senior management is demanded, for, without this commitment, the project cannot in any case succeed. Some of the related information and knowledge to be work out:

i. What are the current industry barriers?

ii. What are the current designer objections?

iii. What are human factors in accepting change?

iv. How to achieve adequate investment?

v. What is the adequate computing technique for KIC?

The motivation for setting up this brief and implementation strategy is to make sure that the application of KIC and its technique will be right, because in the end, it does not depend on the technology alone, but on organization and multi-disciplinary factors. The KIC system must be applied in order to integrate under a design support system. For example, users and system developers must understand what information others need, when they need it, and how to control and distribute it. The KIC implementation, for every stage of engineering design process, can result in either a powerful enabling tool in improving the design process, or a burden of useless and costly complexity. Thus, the KIC integrated design concept, although easy in execution, must be subject to detailed scrutiny considering every aspect of the design activities and life cycle knowledge. In particular, it must be simple to use.

\subsection{Practical Application of a KIC System}

The working system of KIC in the industry should be further improved for commercial use and practical application, rather than remaining as a research tool. This would require the setting up of a strategy, software system and team. We suggest six practical design lessons for the basic and successful application of KIC systems.

i. It is needed to have a framework for underlying structure and classifying the terms for the knowledge as models.

ii. KIC systems must meet the daily needs of users/designers. 
iii. It needs a clear strategy for $\mathrm{KIC}$ to contribute to optimize design and the business.

iv. The application should emphasize on what works, but not perfection. Practically KIC should be focused on knowledge structure, knowledge integration, function specification, desired behavior, and technology.

v. Is necessary to involve senior management in the initiative of KIC application.

vi. Measure the impact of KIC from the very beginning.

One of the successful criteria of KIC system is to solve the problem of knowledge archipelago (islands of knowledge). We point out that it is important to have a strategy, framework and computer-based models to support the practical application of the KIC system. It is because without these, the practical application process will end up in chaos. The computerbased models and the knowledge strategists need to work together. The focus must be getting the right information from the right resources at the right time for the potential users to meet the needs of works. The system must be able to improve the design processes and even can support the business plan. Contribution to the business is one way to market the system by fixing the organization's vision and values with the practical application of KIC. However, it is important to understand that KIC technology is pragmatism and it always has its limits. Thus, the application shall only "focus on what works, not perfection." We recommend that we should emphasize solving the strategic problems in knowledge structure, knowledge integration, function specification, desired behavior, resulting physical states and exploring the enabling technology. In order to apply KIC successfully, it is necessary to have the involvement of senior management, substantial funding and organization reformation or change. This is for removing barriers, making application a priority, and eliminate negative impacts on the gathering of knowledge from multiple disciplinary. Finally, measurement of the impact of KIC application in term of benefit, strength and weakness of the system must be carried out from the beginning. Someone will ultimately question whether benefits are derived from the investment cost on KIC. Thus, it is necessary to monitor the contributions and impacts of KIC applications that can spell out the "advantages" and "proof of the values." Further discussion related to measurement is discussed in the sub-sequence section. 


\subsection{Measuring and Evaluating the Impact of a KIC System: Verification and Validation}

The KIC system must be attested. Verification and validation are the critical evaluation factors of a KIC project. In fact it is equally important to work out these KIC's measurers, as to celebrate the successes of the application. We strongly recommended that the measuring process should be planned from the very beginning of the research and figure out the contributions of the KIC system versus the measurement's factors. There are many other factors in measurement process, however it may be helpful to bear the following two factors in mind: verification and validation. Verification is the process which measures whether the KIC system conforms to the architectural framework and specifications developed as part of the computational methodology (Sriram, 1997). In the verification process, we suggest to measure the correctness and significance of the KIC after the system is implemented properly. Validation is the process which measures whether the implemented KIC system performs the functions that it is intended for (Sriram, 1997). In the validation process, we suggest to measure the application of KIC to see if the system can solve the right problem. Validation in KIC involves setting up criteria for success and measuring the success from these criteria after carefully studying the strength and the weakness features of the system.

The followings are some keys for success or milestone for the measurement phase:

i. Plan the measurement process as early as possible in the development and application of KIC, so that knowledge gathering can be organized around the system and any necessary strategy and technical skills can be appropriated for the research goals.

ii. It is important to be thoroughly familiar with the methods of measurement and must understand how to deviate from them.

iii. Test the contributions of KIC wherever possible using the measurement methods suggested.

iv. Present the finding to the industry experts (or targeting users) as you go so that it is clear what you are claiming.

v. Expose all the measurements to the scrutiny of the industry experts (or targeting users) and review them so as it progresses in order to make the system to stand for the "proof of the values."

vi. Respond to all the doubts expressed by the targeting users and senior management about the contribution of the KIC system, and check whether the measurement methods have the necessary depth for your level of research in KIC.

vii. It is always safe to do more than necessary. 
There are several techniques that can be used in the measurement phase. It is suggested that the performance of the system can be measured up using:

i. Industry as a field trial.

ii. Test design scenario or protocol.

iii. Test design data.

\subsection{Capturing the Life Cycle Knowledge as the Content of KIC}

Future KIC systems must include the life cycle knowledge as the content of the systems, to generate more added-value. These values are, for instance, manufacturability, design for assemble ability, consumer serviceability, recyclability, reusability, and disposal ability. The goal of capturing the life cycle knowledge in KIC systems is to maximize these values in design, while minimizing its costs and environmental impacts. Developing a life cycle knowledge intensive system to support this is a challenging task because of the following characteristics:

i. Large quantity of life cycle knowledge.

ii. Wide diversity of values in life cycle knowledge.

iii. Fragmented relationship within the life cycle knowledge.

iv. Complexity in knowledge-creation from capturing and storing knowledge, to retrieving and reuse of knowledge.

$v$. Usefulness of the knowledge in the life cycle design stages.

vi. Dynamism of life cycle knowledge.

Future research directions in life cycle knowledge for KIC should encounter the following problems and research questions:

i. Systematic methodologies that can support the design easier as well as more complete using the life cycle knowledge.

ii. Implementation issues for the essential life cycle knowledge in a systematic manner.

iii. Where the life cycle starts and ends?

iv. Knowledge life cycle is triggered off by what factors?

v. How to maintain the cyclical and interactive nature of knowledge life cycle?

\section{CONCLUSION}

The papers presented in the KIC-3 workshop provided a vivid discussion on many other themes of interest in knowledge intensive CAD. This paper 
reported an overview of an important discussion from each individual paper. The papers discuss not only theoretical aspects but also practical systems and experiences. Overall, the workshop successfully provided the participants with multiple-disciplinary views of the subject both from computer science and engineering design research angles.

This paper also reported and contributed effectively to the future development and application of knowledge-intensive CAD. More research needs to be done in helping the research design, from life cycle knowledge, theory, implementation strategy, measurement, to practical application. The working system of KIC should be further improved for commercial use, rather than remaining as a research tool. This would require the setting up of a software production system (or team) which would involve considerable financial investment.

\section{REFERENCES}

Brown D.C., Waldron M.B., Yoshikawa H. (1992) Intelligent Computer Aided Design, NorthHolland.

Brown, D.C. (1997) "Which Way to KIC?" Knowledge Intensive CAD, Vol. 2, (eds.) Mäntylä, M., Finger, S, Tomiyama, T., Chapman \& Hall.

Hew, K.P. (1998) A Knowledge-Based Electronic Prototype System (KEPS) for Building and Services Design Integration, Ph.D. Thesis, The University of Reading, UK.

Hew, K.P., Washio, T., Tomiyama, T., Umeda, Y. (1999) "Mathematical Theory of Synthesis Design: Foundation, Framework and Method", Proceeding of $4^{\text {th }}$ International Design Thinking Research Symposium on Design Representation, MIT, April 23-25, 1999, pp. II, 205-217.

Mäntylä, M., Finger, S, Tomiyama, T. (1997) Knowledge Intensive CAD, Vol. 2, Chapman \& Hall.

Mäntylä, M. (1996) "Knowledge-Intensive CAD: Introduction and a Research Agenda", Knowledge Intensive CAD, Vol. 1, (eds.) Tomiyama, T., Mäntylä, M., Finger, S., Chapman \& Hall, 1996.

Sriram, R.D. (1997) Intelligent System for Engineering, Springer.

Tomiyama, T., Mäntylä, M., Finger, S. (1996) Knowledge Intensive CAD, Vol. 1, Chapman \& Hall.

Tomiyama, T. (1998) "An Overview of the Modeling of Synthesis Project", International Symposium on Modeling of Synthesis, The University of Tokyo, Japan, 5 December, 1998, pp. 3-12.

Yoshikawa, H., Gossard, D. (1989) Intelligent CAD I, North-Holland.

Yoshikawa, H., Holden, T. (1990) Intelligent CAD II, North-Holland.

Yoshikawa, H., Arbab, F., Tomiyama, T. (1991) Intelligent CAD III, North-Holland. 